\title{
Expression of trypsin modulating oostatic factor (TMOF) in an entomopathogenic fungus increases its virulence towards Anopheles gambiae and reduces fecundity in the target mosquito
}

\author{
Layla Kamareddine ${ }^{1}$, Yanhua Fan ${ }^{2}$, Mike A Osta $^{1^{*}}$ and Nemat O Keyhani ${ }^{2^{*}}$
}

\begin{abstract}
Background: Adult and larval mosquitoes regulate food digestion in their gut with trypsin modulating oostatic factor (TMOF), a decapeptide hormone synthesized by the ovaries and the neuroendocrine system. TMOF is currently being developed as a mosquitocide, however, delivery of the peptide to the mosquito remains a significant challenge. Entomopathogenic fungi offer a means for targeting mosquitoes with TMOF.
\end{abstract}

Findings: The efficacy of wild type and transgenic Beauveria bassiana strains expressing Aedes aegypti TMOF (Bb-Aa1) were evaluated against larvae and sugar- and blood-fed adult Anopheles gambiae mosquitoes using insect bioassays. Bb-Aa1 displayed increased virulence against larvae, and sugar and blood fed adult A. gambiae when compared to the wild type parent strain. Median lethal dose $\left(\mathrm{LD}_{50}\right)$ values decreased by $\sim 20 \%$ for larvae, and $\sim 40 \%$ for both sugar and blood-fed mosquitoes using Bb-Aa1 relative to the wild type parent. Median lethal time $\left(L T_{50}\right)$ values were lower for blood-fed compared to sugar-fed mosquitoes in infections with both wild type and Bb-Aa1. However, infection using $B b$-Aa1 resulted in $15 \%$ to $25 \%$ reduction in $\mathrm{LT}_{50}$ values for sugar- and blood fed mosquitoes, and $\sim 27 \%$ for larvae, respectively, relative to the wild type parent. In addition, infection with Bb-Aa1 resulted in a dramatic reduction in fecundity of the target mosquitoes.

Conclusions: $B$. bassiana expressing Ae. aegypti TMOF exhibited increased virulence against $A$. gambiae compared to the wild type strain. These data expand the range and utility of entomopathogenic fungi expressing mosquito-specific molecules to improve their biological control activities against mosquito vectors of disease.

Keywords: Trypsin modulating oostatic factor, Biological control, Beauveria bassiana, Anopheles gambiae

\section{Findings}

\section{Background}

Mosquito vectors transmit numerous diseases to humans and animals, causing illness and death that result in huge socio-economical burdens, especially in endemic countries. Control of mosquito vector populations has been almost exclusively based on the use of insecticidal chemicals; however, the strong dependence on insecticides for mosquito control worldwide and the use of such chemicals in

\footnotetext{
*Correspondence: mo07@aub.edu.lb; keyhani@ufl.edu

'Department of Biology, American University of Beirut, Bliss Street, Beirut 11072020, Lebanon

${ }^{2}$ Department of Microbiology and Cell Science, University of Florida, Gainesville, FL 32611, USA
}

agriculture has led to the physiological resistance of important mosquito vectors in recent years [1-3].

Entomopathogenic fungi, such as Metarhizium anisopliae and Beauveria bassiana, both EPA approved biological control agents (http://www.epa.gov/pesticides/), offer an environmentally friendly alternative to chemical insecticides, are virulent to mosquitoes, and have been considered as possible candidates for reducing disease transmission by insect vectors [4-6]. Despite their potential, widespread use of these fungi remains limited, due to a number of factors that include slow killing speed, low resistance to abiotic stress, issues of spore viability, and the amount of material (spores) needed for effective control. Nevertheless, there is still significant interest in using these biological control 
agents due to their effectiveness against insecticide resistant mosquito species [7] and advances in the development of tools and lures for their delivery to mosquitoes [8].

Several attempts at increasing the insecticidal effectiveness of entomopathogenic fungi have been based on the development of recombinant DNA techniques. In this context, transgenic entomopathogenic fungi were developed that express potent insect-specific neurotoxins that kill the infected host [9], or enzymes that enhance fungal resistance to adverse conditions such as UV [10], ultimately increasing spore viability in nature. In addition to increasing their insecticidal potential, entomopathogenic fungal strains can also be manipulated to target the pathogen, e.g. Plasmodium itself within its mosquito vector [11].

Recently, the idea of using targeted host-specific molecules to subvert normal insect development has been proposed $[12,13]$. Insects synthesize hormones and neuropeptides that have been exploited as targets for pest control. Examples of these include diuretic hormones and trypsinmodulating oostatic factors (TMOFs) that are found in insects including mosquitoes and flies. Administration of diuretic hormone increased secretion of fluids from the malphigian tubules leading to water loss and death of the insect. The Ae. aegypti TMOF (Aea-TMOF) was shown to circulate in the hemolymph, bind to gut receptors and inhibit trypsin biosynthesis by exerting a translational control on trypsin mRNA [14]. TMOF is resistant to proteolysis in the gut and easily traverses the gut epithelial cells into the hemolymph in adults and larvae, hence, it can be fed to mosquitoes resulting in inhibition of food digestion, anorexia, and is ultimately lethal to the insect [15-18]. In this report, we measured the virulence of a $B$. bassiana strain expressing Aea-TMOF to adult (sugar and blood-fed) and larval stages of Anopheles gambiae, the major malaria vector in Africa. The effect of this strain on mosquito fecundity was also determined.

\section{Methods}

Anopheles gambiae G3 strain was reared as previously described [19]. Mosquito eggs were treated routinely with 1\% Virkon for 4-5 minutes before floating them, to avoid spread of opportunistic infections in the colony. Wild type and Aea-TMOF expressing (Bb-Aa1) B. bassiana strains [20] were cultured at room temperature on potato dextrose agar (PDA) plates for a period of three to four weeks to allow growth of aerial conidia (spores). To collect spores, the surfaces of two B. bassiana PDA plates were scraped using a sterile cell scraper in the presence of sterile $\mathrm{ddH}_{2} \mathrm{O}$ and the extract was filtered over a glass wool packed column. The flow through material containing spores was centrifuged at $4500 \mathrm{rpm}$ for $10 \mathrm{~min}$ and the pellet was washed twice with $\mathrm{ddH}_{2} \mathrm{O}$. The pellet was resuspended in $\mathrm{dd}_{2} \mathrm{O}$ containing $0.05 \%$ Tween 80 , spores were counted with a Neubauer hemocytometer and adjusted to the appropriate concentrations for mosquito infections.

Adult mosquito bioassays were performed by spraying batches of 45 females (2-days old) each, with spore suspensions of Bb-Aal or the wild type (control) strain and mortality was scored on a daily basis. Briefly, adult mosquitoes were anaesthetized on ice, transferred onto a Whatman paper in a Petri dish, sprayed with the appropriate spore suspension using glass atomizers and then returned back to paper cups. Larval bioassays were conducted in plastic trays $(8.5 \times 13 \times 5.5 \mathrm{~cm})$ each containing 40 three-days old larvae in a total of $110 \mathrm{ml}$ water. Larvae were infected by applying different concentrations of conidia $\left(1.1 \times 10^{9}, 5.5 \times 10^{8}\right.$, $2 \times 10^{8}$ or $1 \times 10^{8}$ conidia in a $50 \mu \mathrm{l}$ olive oil suspension) to the surface of the assay chamber mixed to achieve homogenous surface spreading. The Kaplan-Meier survival test was used to calculate the percent mortality over the indicated time scale. Statistical significance of the observed differences was calculated by the Log-Rank test. Differences were considered to be significant if $P<0.05$. Median lethal time $\left(\mathrm{LT}_{50}\right)$ values for both $B$. bassiana strains were calculated from survival curves of adult mosquitoes (both sugarand blood fed) and larvae infected with $1 \times 10^{8}$ and $5 \times 10^{6}$ spores/ml, respectively, using regression analysis. Statistical analysis of $\mathrm{LT}_{50}$ values was performed using the Student's T-test. Median lethal dose $\left(\mathrm{LD}_{50}\right)$ values were determined using concentrations ranging from $1 \times 10^{7}$ to $2 \times 10^{8}$ spores/ml in adult mosquitoes and $9 \times 10^{5}$ to $1 \times 10^{7}$ spores $/ \mathrm{ml}$ in larvae using the Probit analysis. All experiments were repeated at least three times using different batches of mosquitoes and spores.

To examine the effect of fungal infection on mosquito fecundity, A. gambiae females sprayed with a suspension of $1 \times 10^{8}$ spores $/ \mathrm{ml}$ of wild type or $B b$-Aal strain, were given a blood meal $24 \mathrm{~h}$ after fungal infection. Blood fed females were placed individually into paper cups and eggs were counted $48 \mathrm{~h}$ after blood feeding. Statistical analysis was performed using the Mann Whitney test.

\section{Results and discussion}

B. bassiana strain Bb-Aa1 expresses Aea-TMOF as a fusion protein with a 28 amino acid signal peptide derived from the $B$. bassiana chitinase gene to drive the extracellular secretion of the hormone [20]. Sugar and blood-fed adult, female $A$. gambiae as well as larvae were exposed to spores (conidia) of strain $B b$-Aa1 or the wild type parent to determine the effect of Aea-TMOF expression on virulence. $B b-A a 1$ was more potent than its wild type parent against both sugar and blood-fed adults (Figure 1A and $1 \mathrm{~B})$, causing $40 \%$ reduction in $\mathrm{LD}_{50}$ values $(50 \%$ mortality) in both groups compared to the wild type control (Table 1). However, $\mathrm{LD}_{50}$ values were similar between sugar and blood-fed mosquitoes infected with the same fungal strain, regardless of its type $(B b-A a 1$ or 


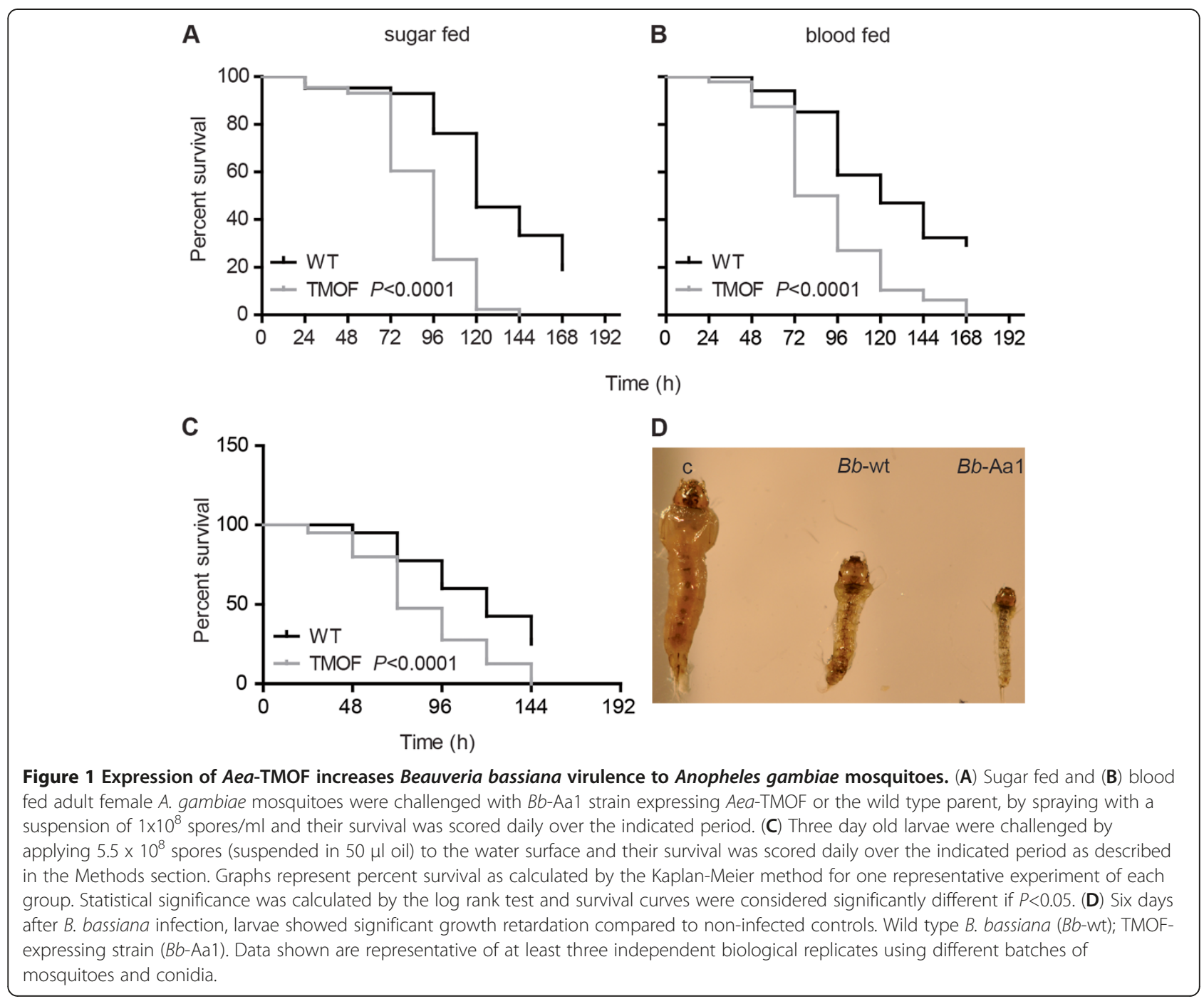

wild type). Infection with $B b$-Aa1 also induced a 15 and $25 \%$ reduction in the mean survival times ( $\mathrm{LT}_{50}$ values) of sugar and blood-fed mosquitoes, respectively, compared to the wild type strain. $\mathrm{LT}_{50}$ values were also lower for blood-compared to sugar fed mosquitoes infected with the same strain, regardless its type. The fact that infections with the same strain, irrespective of its type, resulted in similar $\mathrm{LD}_{50}$ values between sugar and blood-fed mosquitoes, but lowered consistently the $\mathrm{LT}_{50}$ values for the blood- compared to sugar fed group, suggests that the blood meal itself does not seem to affect the virulence of a particular B. bassiana strain but rather mosquito tolerance to infection. Our data are in contrast to Myone et al. ([21]), who reported that blood-fed mosquitoes tended to be similar to or displayed greater median survival times than their unfed cohorts. There are several possible explanations for this discrepancy that can include the assay conditions (Myone et al. used $2 \times 10^{10}$ conidia $/ \mathrm{m}^{2}$ formulated in an oil suspension and sprayed onto sheets) and strain variation (whether fungal or mosquito), and this issue likely deserves greater attention.

A. gambiae larvae infected with $B b$-Aa1 exhibited greater compromised growth and survival as compared to those infected with the wild type strain (Figure 1C). Bb-Aal infection of larvae resulted in 20 and $27 \%$ reductions in $\mathrm{LD}_{50}$ and $\mathrm{LT}_{50}$ values, respectively, compared to the wild type strain (Table 1). Six days after B. bassiana infection, larvae exhibited significant growth retardation evidenced by their abnormally small size compared to non-infected controls; however, the phenotype was more severe in $B b$-Aa1 infected larvae compared to those infected with the wild type strain (Figure 1D).

Egg laying was significantly affected by fungal infection. Infection of $A$. gambiae mosquitoes with the wild-type $B$. bassiana strain resulted in a significant reduction $(\sim 16 \%)$ in fecundity compared to non-infected controls (Figure 2). However, expression of Aea-TMOF resulted in a dramatic reduction $(\sim 60 \%)$ in fecundity compared to controls 
Table $1 \mathrm{LD}_{50}$ and $\mathbf{L T}_{50}$ values of wild type and Aea-TMOF expressing $B b-A a 1$ strain against Anopheles gambiae

$\mathrm{LD}_{50}$ and $\mathrm{LT}_{50}$ values of wild-type and TMOF expressing $B$. bassiana infections in Anopheles gambiae

\begin{tabular}{|c|c|c|c|}
\hline Strain & $\begin{array}{l}\text { Mosquito } \\
\text { stage }\end{array}$ & $\mathrm{LD}_{50}$ (conidia/ml) & $\mathrm{LT}_{50}(\mathrm{~h})$ \\
\hline $\begin{array}{l}\text { Wild-type } B \text {. } \\
\text { bassiana (sf) }\end{array}$ & Adult & $18.34 \pm 2.86 \times 10^{7 a}$ & $133.26 \pm 12.79^{b}$ \\
\hline Bb: spAeaTMOF (sf) & Adult & $\begin{array}{l}11.08 \pm 2.79 \times 10^{7 a} \\
(P=0.01)\end{array}$ & $\begin{array}{l}113.23 \pm 17.56^{b} \\
(P=0.009)\end{array}$ \\
\hline $\begin{array}{l}\text { Wild-type } B . \\
\text { bassiana (bf) }\end{array}$ & Adult & $18.00 \pm 2.01 \times 10^{7 c}$ & $115.04 \pm 11.26^{d}$ \\
\hline Bb: spAeaTMOF (bf) & Adult & $\begin{array}{l}11.31 \pm 0.01 \times 10^{7 c} \\
(P=0.04)\end{array}$ & $\begin{array}{l}86.48 \pm 4.68^{d} \\
(P=0.01)\end{array}$ \\
\hline Wild-type B. bassiana & Larvae & $14.07 \pm 1.24 \times 10^{7 e}$ & $116.31 \pm 13.69^{f}$ \\
\hline Bb: spAeaTMOF & Larvae & $\begin{array}{l}11.32 \pm 0.77 \times 10^{7 e} \\
(P=0.01)\end{array}$ & $\begin{array}{l}85.05 \pm 14.66^{f} \\
(P=0.001)\end{array}$ \\
\hline
\end{tabular}

$\mathrm{a}, \mathrm{c} \mathrm{LD}_{50}$ calculated from the $76 \mathrm{~h}$ time point.

e $\mathrm{LD}_{50}$ calculated from the $48 \mathrm{~h}$ time point using the Probit analysis.

b,d Bioassays performed by spraying mosquitoes with a concentration of $1 \times 10^{8}$ spores $/ \mathrm{ml}$.

${ }^{f}$ Larval bioassays performed using a concentration of $5 \times 10^{6}$ spores $/ \mathrm{ml}$. Statistical analysis was performed used the Student's T-test and values were considered significant if $P<0.05$.

(Figure 2). These data suggest that strain $B b$-Aal also has the potential to reduce the size of $A$. gambiae mosquito populations by severely compromising fecundity.

Aea-TMOF does not induce vertebrate toxicity [22] and has passed EPA approval (http://www.epa.gov/

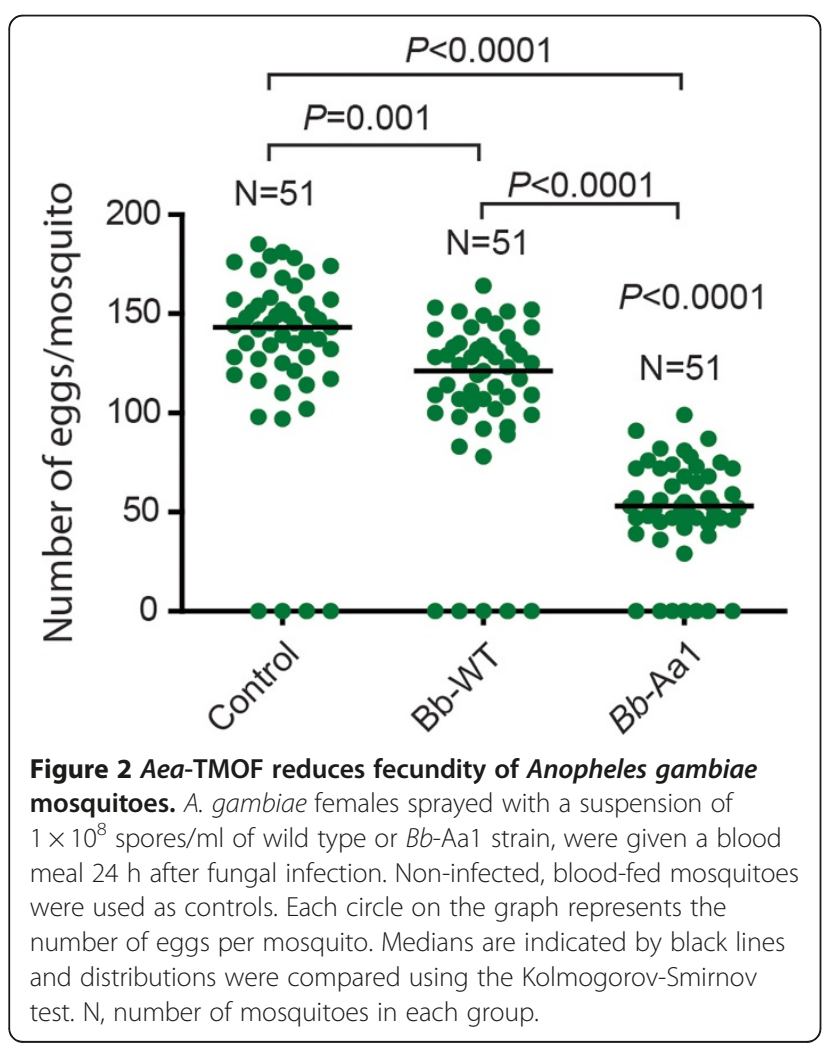

pesticides/). Laboratory bioassays showed that the hormone is effective against all the major species of mosquitoes including anopheline [17]. Data from this as well as a previous study [20] indicate that expressing Aea-TMOF in entomopathogenic fungi is a promising approach for the delivery of the hormone to mosquitoes. Entomopathogenic fungi strongly affect mosquito survival $[4,23,24]$ and may constitute a reliable measure for mosquito control when used as part of an integrated vector management plan. A field trial in a malaria endemic village in Tanzania, using experimental huts that simulate local houses, revealed that certain applications of fungal-treated surfaces lead to the infection of approximately $70 \%$ of mosquitoes entering the hut, which according to their model, is expected to reduce malaria transmission by $75-80 \%$ [25]. Of particular relevance to the data presented here, their model predicts that maintaining a high reduction in malaria transmission rates at lower levels of infected mosquitoes necessitates an increase in fungal virulence [25]. Hence, strains like $B b$-Aal with improved virulence over standard parent strains are expected to strongly reduce malaria transmission even when the prevalence of fungus-infected mosquitoes is moderate.

Fungal strains with a narrower host range and coupled to specific TMOF peptides can also be constructed to maximize specificity and minimize nontarget effects. TMOF is a physiologically important host hormone that must be expressed during the lifestages of adults and larvae to control digestion and growth [26], thus, the mechanisms for resistance to occur can be expected to be life threatening to the mosquito. Despite the fact that TMOF expression increases $B$. bassiana virulence to $A$. gambiae, $\mathrm{LT}_{50}$ values of $B b$-Aal infected mosquitoes suggest that death is not quick enough to completely inhibit females from going through their gonotrophic cycles, even if spores were captured a couple of days before a blood feed. It has been proposed that insecticides exhibiting slow death rates that favor some reproductive success in female mosquitoes can suppress or at least reduce the emergence of resistance [5]. Further, since fungal pathogenesis is a multi-factorial process, where TMOF is not required, but acts to augment virulence, the likelihood of resistance is decreased due to the range of host processes targeted by the fungus, which would have to be overcome. Finally, expression of TMOF does not appear to increase the general virulence of $B$. bassiana to other insects, i.e. Lepidoptera, indicating target specificity [20]. Ideally, TMOF expressing fungi should be incorporated as part of integrated pest management (IPM) programs that do not rely on a single approach for insect control but which utilize compatible and synergistic approaches. 


\section{Conclusions}

Our data show that expression of (Aea)-TMOF in a mycoinsecticide can increase its effectiveness against two important mosquito vectors, Ae. aegypti and A. gambiae. Both survival times and the median lethal dose were lower in the Aea-TMOF expressing strain as compared to the wild type parent. In addition, target fecundity was dramatically reduced. Additional research is needed to examine issues of persistence, delivery, and even greater targeting of hosts, however, the principle of exploiting critical target host molecules for expression in an insect pathogen, which, during infection would compromise the host, can be expanded to a wide range of applications in the biological control of insects.

\section{Competing interests}

The authors declare that they have no competing interests.

\section{Authors' contributions}

MAO and NOK conceived the study. YF constructed the fungal strains, LK performed the experimental work. All authors were involved in data analysis and interpretation. MAO and NOK drafted the manuscript. All authors read and approved the final version.

\section{Acknowledgements}

We thank Hassan Yassine for helping in mosquito rearing and maintenance and Dr. Riyad Sadek for taking the larval photograph. MAO was funded by AUB-URB and The National Center for Scientific Research, Lebanon. NOK was funded by USDA and NSF grants (2010-34135-21095 and IOS-1121392).

Received: 12 September 2012 Accepted: 14 January 2013

Published: 21 January 2013

\section{References}

1. Koekemoer LL, Spillings BL, Christian RN, Lo TC, Kaiser ML, Norton RA, Oliver SV, Choi KS, Brooke BD, Hunt RH, et al: Multiple Insecticide Resistance in Anopheles gambiae (Diptera: Culicidae) from Pointe Noire, Republic of the Congo. Vector Borne Zoonotic Dis 2011, 11(8):1193-1200.

2. Labbe P, Berthomieu A, Berticat C, Alout H, Raymond M, Lenormand T, Weill $M$ : Independent duplications of the acetylcholinesterase gene conferring insecticide resistance in the mosquito Culex pipiens. Mol Biol Evol 2007, 24(4):1056-1067.

3. Lima EP, Paiva MH, de Araujo AP, da Silva EV, da Silva UM, de Oliveira LN, Santana AE, Barbosa CN, de Paiva Neto CC, Goulart MO, et al: Insecticide resistance in Aedes aegypti populations from Ceara. Brazil. Parasit Vectors 2011, 4:5.

4. Blanford S, Chan BH, Jenkins N, Sim D, Turner RJ, Read AF, Thomas MB: Fungal pathogen reduces potential for malaria transmission. Science 2005, 308(5728):1638-1641.

5. Read AF, Lynch PA, Thomas MB: How to make evolution-proof insecticides for malaria control. PLOS Biol 2009, 7(4):e1000058.

6. Scholte EJ, Knols BG, Samson RA, Takken W: Entomopathogenic fungi for mosquito control: a review. J Insect Sci 2004, 4:19.

7. Howard AF, N'Guessan R, Koenraadt CJ, Asidi A, Farenhorst M, Akogbeto M Knols BG, Takken W: First report of the infection of insecticide-resistant malaria vector mosquitoes with an entomopathogenic fungus under field conditions. Malar J 2011, 10:24.

8. Bukhari T, Takken W, Koenraadt CJ: Development of Metarhizium anisopliae and Beauveria bassiana formulations for control of malaria mosquito larvae. Parasites \& vectors 2011, 4:23.

9. Wang C, St Leger RJ: A scorpion neurotoxin increases the potency of a fungal insecticide. Nat Biotechnol 2007, 25(12):1455-1456.

10. Fang W, St Leger RJ: Enhanced UV Resistance and Improved Killing of Malaria Mosquitoes by Photolyase Transgenic Entomopathogenic Fungi. PLoS One 2012, 7(8):e43069.
11. Fang W, Vega-Rodriguez J, Ghosh AK, Jacobs-Lorena M, Kang A, St Leger RJ: Development of transgenic fungi that kill human malaria parasites in mosquitoes. Science 2011, 331(6020):1074-1077.

12. Fan Y, Pereira RM, Kilic E, Casella G, Keyhani NO: Pyrokinin betaneuropeptide affects necrophoretic behavior in fire ants (S. invicta), and expression of beta-NP in a mycoinsecticide increases its virulence. PLOS One 2012, 7(1):e26924.

13. Keyhani NO: Using host molecules to increase fungal virulence for biological control of insects. Virulence 2012, 3(4):415-417.

14. Borovsky D, Rabindran S, Dawson WO, Powell CA, lannotti DA, Morris TJ, Shabanowitz J, Hunt DF, DeBondt HL, DeLoof A: Expression of Aedes trypsin-modulating oostatic factor on the virion of TMV: A potential larvicide. Proc Natl Acad Sci USA 2006, 103(50):18963-18968.

15. Borovsky D: Trypsin-modulating oostatic factor: a potential new larvicide for mosquito control. J Exp Biol 2003, 206(Pt 21):3869-3875.

16. Borovsky D, Carlson DA, Griffin PR, Shabanowitz J, Hunt DF: Mass spectrometry and characterization of Aedes aegypti trypsin modulating oostatic factor (TMOF) and its analogs. Insect Biochem Mol Biol 1993, 23(6):703-712

17. Borovsky D, Meola SM: Biochemical and cytoimmunological evidence for the control of Aedes aegypti larval trypsin with Aea-TMOF. Arch Insect Biochem Physiol 2004, 55(3):124-139.

18. Bylemans D, Borovsky D, Hunt DF, Shabanowitz J, Grauwels L, De Loof A: Sequencing and characterization of trypsin modulating oostatic factor (TMOF) from the ovaries of the grey fleshfly, Neobellieria (Sarcophaga) bullata. Regul Pept 1994, 50(1):61-72.

19. Danielli A, Loukeris TG, Lagueux M, Muller HM, Richman A, Kafatos FC: A modular chitin-binding protease associated with hemocytes and hemolymph in the mosquito Anopheles gambiae. Proc Natl Acad Sci USA 2000, 97(13):7136-7141.

20. Fan Y, Borovsky D, Hawkings C, Ortiz-Urquiza A, Keyhani NO: Exploiting host molecules to augment mycoinsecticide virulence. Nat Biotechnol 2012, 30(1):35-37.

21. Mnyone LL, Kirby MJ, Mpingwa MW, Lwetoijera DW, Knols BGJ, Takken W, Koenraadt CJM, Russell TL: Infection of Anopheles gambiae mosquitoes with entomopathogenic fungi: effect of host age and blood-feeding status. Parasitol Res 2011, 108(2):317-322.

22. Borovsky D: Trypsin modulating oostatic factor for developing resistant crops. In Insecticides design using advanced technologies. Edited by Ishaaya I, Nauen R, Horowitz AR. Berlin Heidelberg: Springer-Verlag; 2007:135-149.

23. Mnyone LL, Kirby MJ, Lwetoijera DW, Mpingwa MW, Knols BG, Takken W, Russell TL: Infection of the malaria mosquito, Anopheles gambiae, with two species of entomopathogenic fungi: effects of concentration, co-formulation, exposure time and persistence. Malaria J 2009, 8:309.

24. Scholte EJ, Ng'habi K, Kihonda J, Takken W, Paaijmans K, Abdulla S, Killeen GF, Knols BGJ: An entomopathogenic fungus for control of adult African malaria mosquitoes. Science 2005, 308(5728):1641-1642.

25. Mnyone LL, Lyimo IN, Lwetoijera DW, Mpingwa MW, Nchimbi N, Hancock PA, Russell TL, Kirby MJ, Takken W, Koenraadt CJ: Exploiting the behaviour of wild malaria vectors to achieve high infection with fungal biocontrol agents. Malaria J 2012, 11:87

26. Borovsky D: Biosynthesis and control of mosquito gut proteases. IUBMB Life 2003, 55(8):435-441.

doi:10.1186/1756-3305-6-22

Cite this article as: Kamareddine et al:: Expression of trypsin modulating oostatic factor (TMOF) in an entomopathogenic fungus increases its virulence towards Anopheles gambiae and reduces fecundity in the target mosquito. Parasites \& Vectors 2013 6:22 